\title{
Paidéia: Relatório de Gestão - 2012 ${ }^{1}$
}

\author{
Manoel Antônio dos Santos \\ Universidade de São Paulo, Ribeirão Preto-SP, Brasil
}

Neste Relatório de Gestão apresentamos indicadores que conferem visibilidade à gestão editorial da Paidéia no ano de 2012. Com a publicação desse documento preservamos a tradição de apresentar à comunidade científica um balanço do que a revista produziu no último ano, tornando explícito o modus operandi do processo de tramitação dos manuscritos no período.

A divulgação da produção científica é lastreada em um rigoroso processo de avaliação por pares. Ao sistematizar informações relativas ao processo editorial, é possível localizar a contribuição da Paidéia no cenário contemporâneo das publicações científicas na área das Ciências Humanas, especialmente da Psicologia.

Este relatório está subdividido nas seguintes seções: Política editorial e critérios de arbitragem, Processo editorial - 2012 e Considerações finais.

\section{Política editorial e critérios de arbitragem}

A Paidéia é uma publicação quadrimestral do Programa de Pós-graduação em Psicologia da Faculdade de Filosofia, Ciências e Letras de Ribeirão Preto da Universidade de São Paulo - FFCLRP-USP. Tem como escopo e foco editorial publicar estudos relacionados à Psicologia. Os artigos publicados devem enquadrar-se nas categorias: relato de pesquisa, estudo teórico e revisão sistemática da literatura. Até 2011 eram admitidas as seguintes categorias de manuscritos: relato de pesquisa, estudo teórico, relato de experiência profissional, revisão sistemática da literatura, comunicação breve, nota técnica e resenha. Essas categorias estão refletidas nos resultados divulgados no presente relatório.

Em 2013, a Paidéia completa 22 anos de existência. Desde sua fundação, em agosto de 1991, foram publicados 53 fascículos, do volume 1 ao 23, e seu acervo encontra-se totalmente disponibilizado, em acesso livre e gratuito assegurado pela Coleção SciELO. Os fascículos primam pela diversidade e abrangência dos temas, bem como pela pluralidade de delineamentos metodológicos contidos em seus artigos. A linha editorial é generalista, em respeito à diversidade teórico-metodológica, epistemológica e temática que permeia a área da Psicologia.

Em termos da classificação no sistema Qualis Periódico, na área da Psicologia (Comissão Qualis Periódico da Psicologia, 2012), a Paidéia ascendeu do estrato B1, no qual se encontrava até 2011, para A1, que é a posição mais elevada do ranking da avaliação, o que evidencia o reconhecimento do

\footnotetext{
${ }^{1}$ Endereço para correspondência:

Prof. Dr. Manoel Antônio dos Santos. Universidade de São Paulo. Faculdade de Filosofia, Ciências e Letras de Ribeirão Preto. Departamento de Psicologia. Av. Bandeirantes, 3900. CEP: 14040-901. Ribeirão PretoSP, Brasil.E-mail: masantos@ffclrp.usp.br
}

lugar destacado que esse periódico vem ocupando no conjunto das publicações científicas brasileiras. Esse nível de excelência também ficou patenteado por outro indicador importante - no início de 2012 a revista foi uma das duas publicações nacionais contempladas com o Programa de Apoio da CAPES para alavancar a internacionalização dos periódicos científicos na área da Psicologia. Essa indicação foi patrocinada pela Comissão de Avaliação Qualis Periódicos e pela Coordenação de Área de Avaliação (Psicologia) da CAPES. O Qualis A1 e o fato de ter sido escolhida pela CAPES para fazer jus ao Programa de Apoio a periódicos com potencial para internacionalização são conquistas que consagraram a Paidéia como uma das principais revistas no panorama atual da Psicologia.

O prestígio angariado pela Paidéia junto à comunidade científica se deve à sua seletiva política de divulgação do conhecimento de qualidade. A aferição rigorosa e criteriosa tem sido sustentada com o apoio de um Conselho Editorial altamente qualificado, estruturado em função das áreas e dos temas abordados nos artigos submetidos à apreciação. Esse Conselho é constituído por nomes expressivos da comunidade acadêmica, tanto no âmbito nacional como internacional. A tramitação dos manuscritos está a cargo de uma Comissão Editorial constituída por Editores Associados, em sua maioria docentes vinculados ao Programa de Pós-graduação em Psicologia da FFCLRP-USP. Essa Comissão coordena o trabalho rigoroso de revisão de pares, lançando mão de consultores ad hoc com reconhecida competência, que têm a missão de avaliar e aprimorar os manuscritos submetidos à avaliação.

Para consolidar sua visibilidade no plano nacional e internacional, a Paidéia está indexada em 13 bases (ou portais de texto completo) nacionais e internacionais, a saber: SciELO - Scientific Eletronic Library Online (FAPESP/BIREME), Scopus (Elsevier), PsycINFO - Psychological Abstracts (American Psychological Association), PASCAL - L'Institut de l'Information Scientifique et Technique (INIST), LILACS Literatura Latino-Americana e do Caribe em Ciências da Saúde (Centro Latino-americano y de Caribe de Informaciones em Ciências de la Salud), CLASE - Citas Latinoamericanas en Ciências Sociales y Humanidades (Universidad Nacional Autónoma de México), PSICODOC (Colégio Oficial de Psicólogos de Madrid / Universidad Complutense de Madrid), DOAJ - Directory of Open Access Journals, Ulrich's International Periodicals Directory, Catálogo Latindex, IRESIE (Universidad Nacional Autónoma de México), Index-Psi Periódicos (CFP/PUCCAMP) e REDALYC - Red de Revistas Científicas de América Latina y el Caribe, España y Portugal.

A submissão dos manuscritos é inteiramente informatizada, por meio do sistema eletrônico Submission, 
disponibilizado pela SciELO. A revista adota o sistema de avaliação blind review, que preserva a identidade dos autores e dos avaliadores. Os textos submetidos à apreciação devem enquadrar-se, prioritariamente, na categoria relato de pesquisa. Em cada fascículo são publicados cerca de 13 artigos originais de pesquisa. A partir de 2012 não são mais admitidos relatos de experiência profissional, comunicação breve, nota técnica e resenha de livro. Isso porque a Comissão Editorial decidiu, como parte da nova política editorial da revista, investir maciçamente na captação de artigos que dêem visibilidade a resultados de pesquisas derivados de estudos empíricos originais, com delineamento quanti ou qualitativo. O potencial de gerar impacto na área de conhecimento também é um critério utilizado para seleção dos manuscritos para publicação. Para serem selecionados para publicação os manuscritos devem ser de interesse internacional e não apenas local.

No que concerne às fontes de financiamento, a Paidéia tem contado com aportes de recursos financeiros captados anualmente do Programa de Apoio às Publicações Científicas Periódicas da USP e do Programa de Apoio a Publicações Científicas do CNPq, além do apoio do Programa de Pósgraduação em Psicologia da FFCLRP-USP.

Ao final de cada fascículo são incluídas as normas de publicação, que estão disponíveis online nosidiomas: português, inglês e espanhol. Essas normas (Instruções aos Autores) estão disponibilizadas na página da SciELO (http://www.scielo.br/paideia). O último fascículo de cada volume contempla a relação de assessores ad hoc, que durante o ano vigente contribuíram com a imprescindível tarefa de revisão dos manuscritos.

A versão impressa da Paidéia é distribuída gratuitamente para, aproximadamente, 160 bibliotecas de universidades públicas brasileiras vinculadas à Rede Brasileira de Bibliotecas da Área de Psicologia - ReBAP. Desse modo, os fascículos são disponibilizados para cursos de graduação em Psicologia, bem como alguns cursos de Educação, além de países do exterior, como Portugal, Espanha, México, França, Reino Unido e EUA. Isso significa que, três vezes ao ano, são enviados exemplares para instituições de nível superior de grande parte dos estados brasileiros e algumas do exterior, bem como para Programas de Pós-graduação nas áreas de Psicologia ou Educação. Também são mantidas permutas com periódicos brasileiros e internacionais da área de Psicologia e Saúde. O último exemplar de cada volume também é encaminhado a todos os consultores ad hoc que colaboraram com a revista durante 0 ano, em reconhecimento à colaboração oferecida na emissão de pareceres. Nesse último fascículo (versão impressa) de cada volume disponibilizamos a relação dos consultores ad hoc que colaboraram com a revista durante o ano vigente. É uma forma de agradecimento aqueles que contribuíram com a revisão dos manuscritos submetidos à apreciação da revista.

No que concerne ao processo de arbitragem dos manuscritos, a Paidéia adota o mecanismo de revisão cega e dupla por pares de reconhecido saber e prestígio junto à comunidade científica. Assim, as identidades dos autores e dos assessores ad hoc são mantidas no mais absoluto sigilo.
Os textos submetidos à avaliação devem estar de acordo com as orientações das normas de publicação e podem ser aceitos ou recusados pela Comissão Editorial, com base nas recomendações de seus membros e dos consultores ad hoc designados para aprimorar o processo avaliativo.

Em relação à disponibilidade dos conteúdos, a Paidéia adota a Licença Creative Commons, CC BY-NC Atribuição não comercial. Com essa licença é permitido acessar, baixar (download), copiar, imprimir, compartilhar, reutilizar e distribuir os artigos, desde que para uso não comercial e com a citação da fonte, conferindo os devidos créditos autorais à revista. Nesses casos, nenhuma permissão é necessária por parte dos autores ou dos editores.

O acervo completo (fascículos antigos) da revista encontra-se disponibilizado no site da SciELO, a partir da conversão dos arquivos para o padrão utilizado por esta base de dados, ampliando assim a visibilidade dos artigos publicados, em conformidade com a política de acesso livre e universal aos conteúdos da revista.

Outro aspecto que diferencia a Paidéia no cenário das publicações da área de Psicologia é a disponibilização sistemática e periódica de informações sobre o processo de gestão do periódico. No primeiro fascículo de cada volume/ano publicamos um Relatório de Gestão, no qual buscamos dar visibilidade e transparência ao trabalho consolidado no ano anterior. Esse documento contém indicadores objetivos que permitem aferir o fluxo da revista, como o tempo de tramitação dos manuscritos, a procedência dos autores que têm seus artigos publicados, a origem institucional dos revisores/consultores ad hoc, entre outros tópicos de interesse (Santos, 2010, 2011, 2012).

\section{Processo editorial - 2012}

Ao considerar o processo editorial da Paidéia no ano de 2012, observa-se que o tempo médio de tramitação dos manuscritos foi de, aproximadamente, dez meses. Neste ano, a revista recebeu 159 manuscritos novos, dos quais 48 foram aprovados, 80 recusados e 31 ainda estavam em processo de avaliação em Janeiro de 2013, quando da redação deste relatório (Tabela 1). Considerando os manuscritos cujo processo editorial foi encerrado em $2012(n=128)$, a revista aprovou $37,5 \%$ e recusou $62,5 \%$.

Tabela 1

Situação Editorial dos Manuscritos Submetidos à Revista Paidéia em 2012

\begin{tabular}{ll}
\hline Parecer final & $\boldsymbol{n}$ \\
\hline Recebidos (TOTAL) & 159 \\
Aprovados & 48 \\
Recusados & 80 \\
Em processo editorial & 31 \\
\hline
\end{tabular}

Tempo entre submissão e publicação em 2012 = 10 meses

No ano de 2012 houve aumento expressivo no número de manuscritos submetidos pelo Submission. Esses manuscritos foram enquadrados nas seguintes categorias: (a) Relato de 
pesquisa (82\%); (b) Estudo teórico (9,3\%); (c) Revisão sistemática da literatura (6,8\%), (d) Resenha (1,9\%), assim como pode ser observado na Tabela 2.

Tabela 2

Categoria dos Manuscritos Submetidos à Paidéia em 2012

\begin{tabular}{lcc}
\hline Tipo de manuscrito & $\boldsymbol{n}$ & $\mathbf{\%}$ \\
\hline Relato de Pesquisa & 132 & 82,0 \\
Estudo Teórico & 15 & 9,3 \\
Revisão Sistemática da Literatura & 11 & 6,8 \\
Resenha & 3 & 1,9 \\
Total & 161 & 100 \\
\hline
\end{tabular}

Em 2012, no volume 22 (fascículos 51, 52 e 53), foram publicados 42 artigos, três resenhas, além de três editoriais e um relatório de gestão. As publicações enquadraram-se nas seguintes categorias: (a) Relato de pesquisa (71,4\%); (b) Estudo teórico (11,9\%); (c) Revisão sistemática da literatura (9,5\%); (d) Resenha (7,2\%), como evidencia a Tabela 3.

Tabela 3

Categoria dos Manuscritos Publicados na Paidéia em 2012

\begin{tabular}{lcc}
\hline Tipo de manuscrito & $\boldsymbol{n}$ & $\mathbf{\%}$ \\
\hline Relato de pesquisa & 30 & 71,4 \\
Estudo teórico & 5 & 11,9 \\
Revisão Sistemática da Literatura & 4 & 9,5 \\
Resenha & 3 & 7,2 \\
Total & 42 & 100 \\
\hline
\end{tabular}

A Figura 1 apresenta a evolução da quantidade de manuscritos submetidos e seu destino no triênio 2010-2012. Em 2012, como referido houve aumento no número de manuscritos submetidos, assim como no montante de trabalhos recusados, o que denota que tem havido reconhecimento crescente da revista por parte da comunidade científica, comitantemente ao incremento do rigor na análise dos artigos.

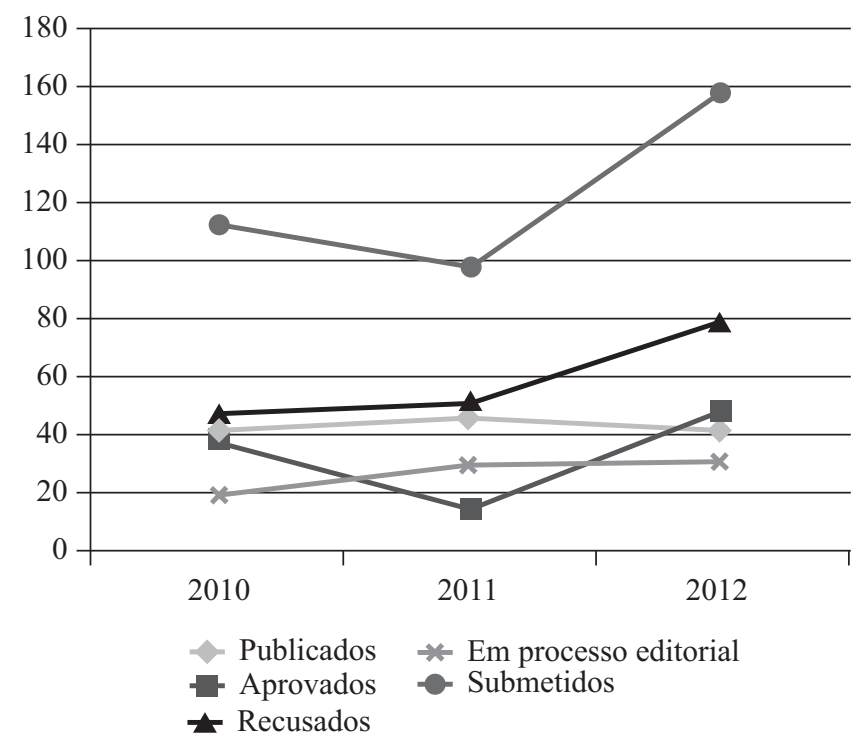

Figura 1. Número de Manuscritos Submetidos à Paidéia e seu Desfecho Editorial no Triênio 2010-2012
No que concerne à filiação institucional dos autores, a Tabela 4 mostra que a Paidéia publicou artigos provenientes de 27 universidades/faculdades/institutos brasileiros, com discreto predomínio da Universidade de Brasília. Houve um razoável aumento na quantidade de artigos com autores de filiação institucional internacional, principalmente de Portugal. Nota-se que os autores dos artigos publicados estão vinculados a uma multiplicidade de instituições de nível superior, de caráter público, privado e confessional, de diversos estados da federação brasileira e do exterior. Houve predomínio de universidades federais em relação às estaduais.

Tabela 4

Origem Institucional dos Autores Cujos Artigos Foram Publicados na Paidéia em 2012

\begin{tabular}{|c|c|c|}
\hline Instituições nacionais & $n$ & $\%$ \\
\hline Universidade de Brasília - DF & 18 & 19,5 \\
\hline Universidade Federal do Rio Grande do Sul - RS & 14 & 15,0 \\
\hline Pontifícia Universidade Católica - SP, MG, RS, GO & 7 & 7,6 \\
\hline Universidade Federal de São Carlos - SP & 6 & 6,5 \\
\hline Universidade São Francisco - SP & 4 & 4,3 \\
\hline Universidade Federal de São Paulo - SP & 4 & 4,3 \\
\hline Universidade Federal da Paraíba - PB & 4 & 4,3 \\
\hline Universidade Estadual de Campinas - SP & 3 & 3,3 \\
\hline Universidade Católica do Rio de Janeiro - RJ & 3 & 3,3 \\
\hline Universidade Estadual Paulista - SP & 3 & 3,3 \\
\hline Universidade de São Paulo - SP & 3 & 3,3 \\
\hline Universidade Estadual de Londrina - PR & 3 & 3,3 \\
\hline Universidade Federal da Bahia - BA & 2 & 2,2 \\
\hline Universidade do Estado do Rio de Janeiro - RJ & 2 & 2,2 \\
\hline Universidade Federal do Triângulo Mineiro - MG & 2 & 2,2 \\
\hline Universidade Federal do Espírito Santo - ES & 2 & 2,2 \\
\hline Universidade Federal de Juiz de Fora - MG & 2 & 2,2 \\
\hline Universidade Federal de Santa Catarina - SC & 1 & 1,1 \\
\hline Universidade Estadual do Rio de Janeiro - RJ & 1 & 1,1 \\
\hline Universidade Federal de Sergipe - SE & 1 & 1,1 \\
\hline Faculdade de Filosofia, Ciências e Letras de Caruaru - PE & 1 & 1,1 \\
\hline Faculdades Integradas de Jaú - SP & 1 & 1,1 \\
\hline Universidade Federal de Pernambuco - PE & 1 & 1,1 \\
\hline Universidade Federal da Grande Dourados - MS & 1 & 1,1 \\
\hline Universidade Presbiteriana Mackenzie - SP & 1 & 1,1 \\
\hline Universidade de Passo Fundo - RS & 1 & 1,1 \\
\hline Universidade Federal do Ceará - CE & 1 & 1,1 \\
\hline Total & 92 & 100 \\
\hline Instituições internacionais & $n$ & $\%$ \\
\hline Universidade Católica Portuguesa, Portugal & 8 & 47,0 \\
\hline Universidade de Porto, Portugal & 4 & 23,5 \\
\hline Univerdidade de Aveiro, Portugal & 2 & 11,8 \\
\hline Univerdidade do Minho, Portugal & 1 & 5,9 \\
\hline University of Stavanger, Noruega & 1 & 5,9 \\
\hline Universidade de Cambridge, Inglaterra & 1 & 5,9 \\
\hline Total & 17 & 100 \\
\hline
\end{tabular}


No que concerne à procedência dos consultores ad hoc, nota-se o predomínio de revisores lotados em universidades do Estado de São Paulo (61,2\%) que, somados aos avaliadores oriundos de outras instituições pertencentes à região Sudeste, totalizaram $70,6 \%$ do corpo de revisores. A região Sul comportou $11,7 \%$ dos assessores e a região Nordeste obteve uma participação de $8,2 \%$. A região Centro-Oeste obteve um total de $3,5 \%$ e a região Norte $1,2 \%$. Nota-se, ainda, uma quantidade significativa de avaliadores internacionais, 4,8\% (Tabela 5).

Tabela 5

Abrangência do Corpo de Consultores ad hoc da Paidéia Conforme Origem Geográfica dos Avaliadores $(n=85)$

\begin{tabular}{|c|c|c|}
\hline \multirow{2}{*}{$\begin{array}{l}\text { Internacionais } \\
\text { País }\end{array}$} & \multicolumn{2}{|c|}{ Consultores } \\
\hline & $n$ & $\% *$ \\
\hline Estados Unidos & 1 & 1,2 \\
\hline Reino Unido & 1 & 1,2 \\
\hline França & 1 & 1,2 \\
\hline Canadá & 1 & 1,2 \\
\hline Subtotal & 4 & 4,8 \\
\hline Nacionais & \multicolumn{2}{|c|}{ Consultores } \\
\hline Estado & $n$ & $\%{ }^{*}$ \\
\hline \multicolumn{3}{|l|}{ Região Sudeste } \\
\hline São Paulo & 52 & 61,2 \\
\hline Rio de Janeiro & 4 & 4,7 \\
\hline Minas Gerais & 4 & 4,7 \\
\hline Subtotal & 60 & 70,6 \\
\hline \multicolumn{3}{|l|}{ Região Sul } \\
\hline Rio Grande do Sul & 4 & 4,7 \\
\hline Paraná & 4 & 4,7 \\
\hline Santa Catarina & 2 & 2,3 \\
\hline Subtotal & 10 & 11,7 \\
\hline \multicolumn{3}{|l|}{ Região Nordeste } \\
\hline Pernambuco & 3 & 3,5 \\
\hline Bahia & 2 & 2,3 \\
\hline Ceará & 1 & 1,2 \\
\hline Rio Grande do Norte & 1 & 1,2 \\
\hline Subtotal & 7 & 8,2 \\
\hline \multicolumn{3}{|l|}{ Região Centro-Oeste } \\
\hline Distrito Federal & 2 & 2,3 \\
\hline Goiás & 1 & 1,2 \\
\hline Subtotal & 3 & 3,5 \\
\hline \multicolumn{3}{|l|}{ Região Norte } \\
\hline Pará & 1 & 1,2 \\
\hline Subtotal & 1 & 1,2 \\
\hline
\end{tabular}

${ }^{*}$ Cálculo baseado no total de 85 assessores que colaboraram com a revista em 2012.

A Figura 2 mostra a distribuição percentual dos consultores ad hoc segundo a procedência geográfica.

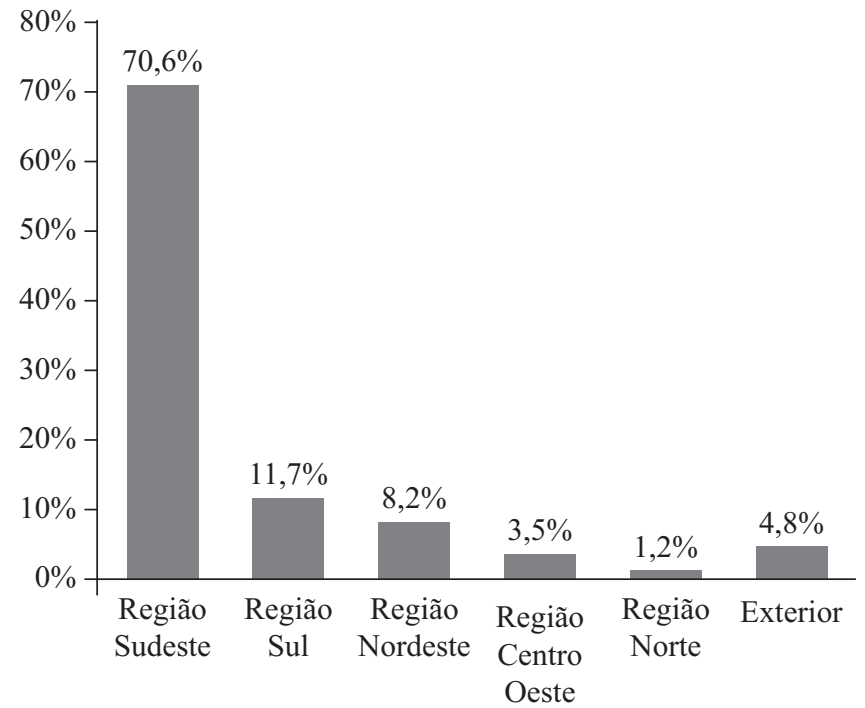

Figura 2. Distribuição dos Consultores ad hoc que Colaboraram com a Paidéia em 2012 em função da Região de Origem.

\section{Considerações Finais}

Este Relatório de Gestão sistematiza informações que permitem mapear o quadro da tramitação editorial dos manuscritos submetidos à apreciação da Paidéia durante o ano de 2012. A comparação dos dados acumulados no último triênio mostra que a revista vem fortalecendo seu processo editorial de forma gradual e consistente, consolidando-se como espaço aglutinador do conhecimento produzido no campo da Psicologia. No entanto, existem fragilidades que precisam ser consideradas, tais como a morosidade no processo de avaliação dos manuscritos (que em parte tem sido superada com as mudanças implementadas no processo de submissão, que passou a ser totalmente online pelo sistema da Scielo), o baixo percentual de artigos de autores estrangeiros e a excessiva concentração de consultores ad hoc em estados da Região Sudeste. A superação desses desafios demanda adoção de estratégias específicas.

Um aspecto positivo a ser destacado no presente relatório é a introdução da publicação da versão completa dos artigos em inglês visando a maior visibilidade internacional da revista. Outro ponto positivo é que o índice de "endogenia” da revista (artigos oriundos de autores da Universidade de São Paulo) continua baixo - da ordem de 3,3\% dos artigos publicados em 2012. Esse índice era de 9,6\% em 2011 (Santos, 2012), 9,7\% em 2010 (Santos, 2011) e 18,0\% em 2009 (Santos, 2010). Também houve aumento de artigos de origem internacional em comparação com o ano de 2011.

Como resultado dos esforços continuados que têm sido empreendidos sistematicamente ao longo dos últimos anos, a Paidéia hoje ocupa um lugar destacado entre as publicações da área da Psicologia. Estamos entre as três revistas nacionais da área classificadas como A1 (Comissão Qualis Periódico da Psicologia, 2012). A implantação da submissão online foi uma condição essencial para organizar e gerenciar o fluxo de informações, bem como agilizar o 
tempo de publicação, que ainda está aquém do desejável e deve ser aprimorado. São necessários investimentos para aumentar a visibilidade da revista no âmbito internacional, ampliar a indexação em bases internacionais e aprimorar os indicadores bibliométricos.

Quando se fala em ciência comunicada, é preciso lembrar que a publicação pode se dar por diversos meios, com destaque para os periódicos científicos, devido ao controle de qualidade, maior credibilidade, regularidade e visibilidade da informação. O conhecimento científico é, essencialmente, oriundo de pesquisa original, submetida à avaliação por pares (peer review process) e publicada em revista indexada, com atenção à análise de uso e impacto da informação veiculada. A indexação em bases especializadas ou multidisciplinares é o selo de qualidade de uma revista.

A questão do idioma pátrio, privilegiado na publicação, continua sendo um entrave para o incremento da visibilidade dos artigos editados pelos periódicos nacionais. Há anos a Paidéia vem publicando resumos em português, inglês e espanhol, o que sempre se constituiu em um diferencial em relação a outras revistas da área da Psicologia, que tradicionalmente só exigem o resumo em português e inglês. Em 2012 implementamos a publicação bilíngue dos artigos, ou seja, disponibilizamos na biblioteca eletrônica (SciELO) as versões completas em português e em inglês, seguindo o caminho aberto por revistas de outras áreas do conhecimento. A partir do segundo fascículo de 2013, os artigos serão publicados apenas em inglês, tanto na versão impressa como na versão online. Desse modo cumprimos a meta estabelecida para que a produção do conhecimento em Psicologia possa alcançar os patamares de excelência desejáveis e se perfilar com outras áreas mais avançadas.

Considerando o incremento considerável da produção científica nacional nos últimos anos, fruto de uma política de estímulo que muitas vezes é confundida com o deletério produtivismo resultante da pressão por publicar, é altamente desejável que as revistas nacionais possam dar maior transparência aos seus processos de publicação. Afinal, os periódicos científicos cumprem um papel destacado no processo de disseminação do conhecimento produzido. São os principais meios de publicação e também os de maior prestígio e credibilidade.

\section{Referências}

Comissão Qualis Periódico da Psicologia (2012). Comunicado $n^{\circ}$ 0001/2012 - Área de Psicologia: Atualização do webqualis da área. Brasília: Coordenação de Aperfeiçoamento de Pessoal de Nível Superior. Recuperado em 30 de março 2012, de http://www.capes. gov.br/images/stories/download/avaliacao/Qualis_-_ Psicologia.pdf

Santos, M. A. (2010). Paidéia: relatório de gestão - 2009. Paidéia, 20(45), 3-6.

Santos, M. (2011). A. Paidéia: relatório de gestão - 2010. Paidéia, 21(48), 5-8.
Santos, M. (2012). A. Paidéia: relatório de gestão - 2011. Paidéia,22(51), 5-9.

Manoel Antônio dos Santos é Professor Associado 3 da Faculdade de Filosofia, Ciências e Letras de Ribeirão Preto da Universidade de São Paulo, Editor da Paidéia.
Como citar este documento:

Santos, M. A. (2013). Paidéia: Relatório de Gestão - 2012. Paidéia (Ribeirão Preto), 23(54), 3-7. doi:http://dx.doi.org/10.1590/1982-43272354201302 


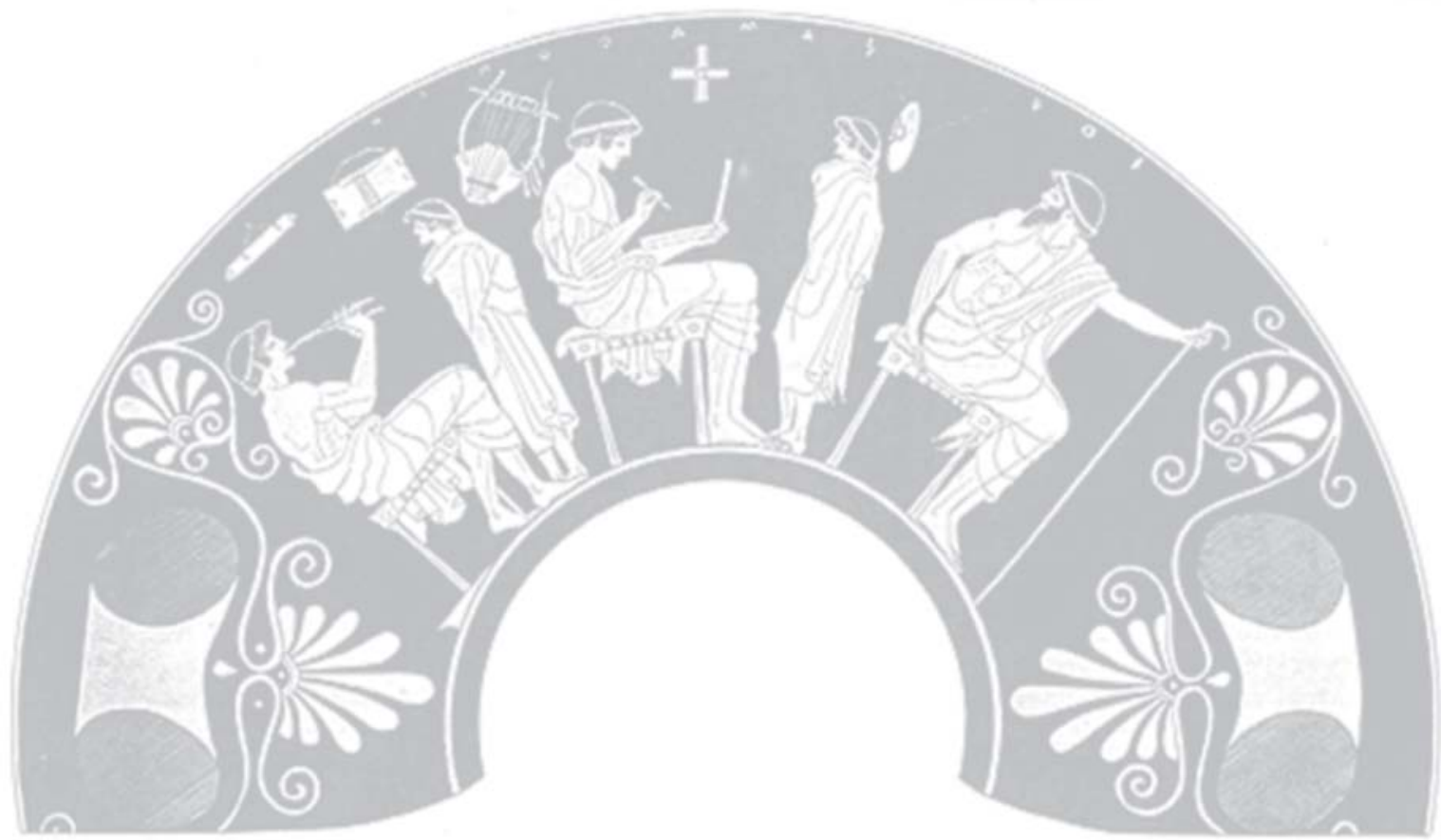

